\title{
Ultrasound screening in a neonatal unit
}

\section{HM Swanepoel', F Ismail'}

${ }^{1}$ Clinical Head, Ultrasound, Department of Radiology, Steve Biko Academic Hospital, University of Pretoria, Pretoria, South Africa 2Department of Radiology, Steve Biko Academic Hospital, University of Pretoria, Pretoria, South Africa

Ultrasound (US) is an important imaging tool for evaluating the neonate. US is portable and non-invasive and can be repeated as often as is necessary.

It is therefore the imaging modality of choice for imaging the critically ill neonate.

The quality of US and its diagnostic accuracy depends on many factors; suitable equipment with appropriate settings and probes, scanning at appropriate times and the experience and expertise of the examiners.

This is a brief review of the typical US imaging findings encountered in a neonatal unit.

\section{Cranial ultrasound}

The majority of requests for US in the neonatal unit are for cranial examinations. There is no consensus as to the optimal times for doing cranial US. It varies with the gestational age of the neonate, expected pathologies and clinical picture. Days 3-5, 10-14, about day 28 and pre-discharge if uncomplicated, or only day 3-5 and then pre-discharge, others see week 3640 postmenstrual age as optimal. ${ }^{2}$

\section{Indications for cranial ultrasound in a neonatal unit}

1) Prematurity (the main indication)

2) US as part of the routine antenatal care; US should be done in the neonatal unit to confirm abnormalities detected antenatally.

3) Brain injuries occurring at birth or shortly thereafter

4) Neonates with congenital or acquired infections, coagulopathies, metabolic or neurological abnormalities.

\section{Method}

Access is through the anterior and posterior fontanels, the mastoid foramen and poorly ossified parts of the temporal bone. ${ }^{2}$

Cranial Ultrasound should start with a coronal sweep through the anterior fontanel. This gives a valuable general impression of normal and abnormal structures (Fig l). Turn the transducer $90^{\circ}$ to a sagittal orientation and sweep from one side to the other. Evaluate the ventricular system, choroid plexus and midline structures such as the corpus callosum, third and fourth ventricles, as well as the thalami and caudate
Figure 1: Premature brain. Normal coronal section. Note anterior horns of the lateral ventricles (long arrows) and prominent cavum septum pellucidum (short arrow)

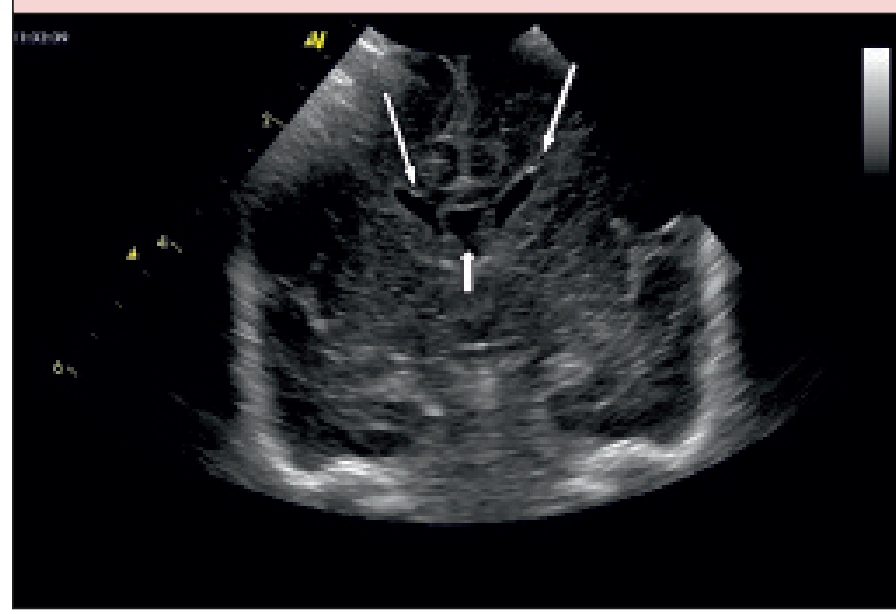

nuclei (Fig 2). The cerebellum is better demonstrated through the mastoid view. The transtemporal view is used when utilizing Doppler ultrasound. ${ }^{3}$

Figure 2: Normal saggital view, demonstrating the corpus callosum -short arrow, and cavum septum pellucidum long arrow.

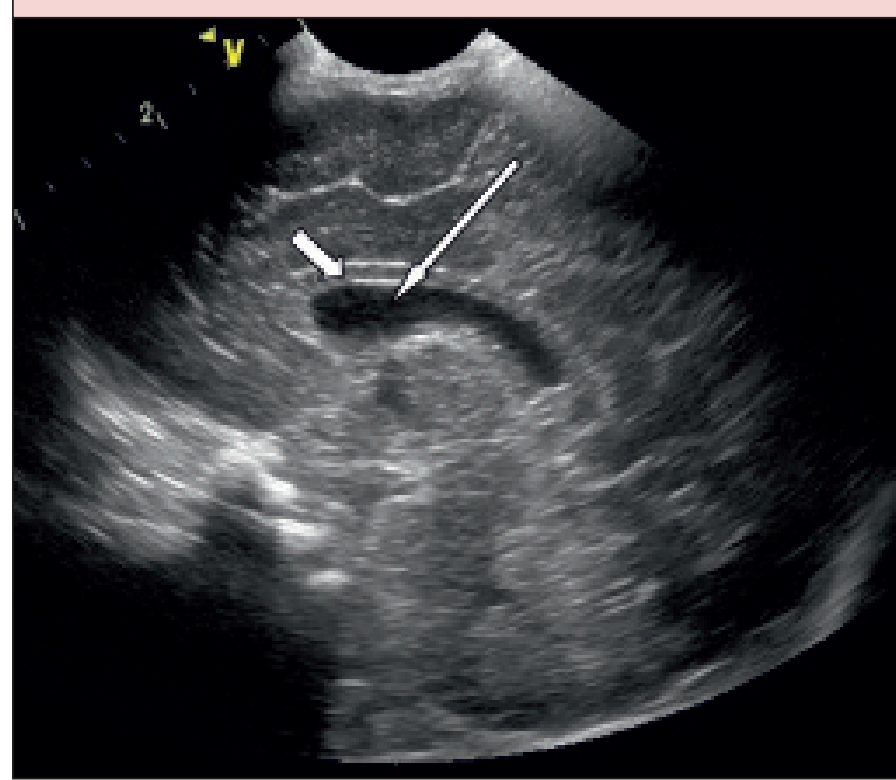




\section{Interpretation}

There are differences between the immature preterm and the mature full term brain. In preterm infants, the ventricular system is wider; there is a large cavum septum pellucidum and cavum vergae, less gyration and increased echogenicity of the periventricular white matter. In the mature brain, the ventricles are smaller, gyration is pronounced and parenchymal differentiation increases. ${ }^{1}$ Doppler in the premature brain has a low diastolic velocity and higher resistance index (RI) value than in the mature brain.

\section{Conditions that can be demonstrated on cranial ultrasound}

\begin{tabular}{|l|l|}
\hline Brain haemorrhage & $\begin{array}{l}\text { Germinal matrix haemorrhages (GMH) } \\
\text { Intraventricular heamorrhages (IVH) } \\
\text { Intraparenchymal heamorrhages / } \\
\text { Periventricular heamorrhages (PVH)/ } \\
\text { Periventricular heamorrhagic infarction (PVHI) } \\
\text { Epidural (EDH), Subdural (SDH), } \\
\text { Subarachnoid haemorrhage (SAH) }\end{array}$ \\
\hline Hypoxic-ischemic & $\begin{array}{l}\text { HIE in premature infants } \\
\text { HIE in term infants }\end{array}$ \\
\hline Neonatal focal infarction & $\begin{array}{l}\text { Ventriculomegaly } \\
\text { Agenesis of the corpus callosum } \\
\text { Holoprosencephaly (Alobar, lobar, semilobar } \\
\text { and septo-optic dysplasia) } \\
\text { Chiari malformation } \\
\text { Posterior fossa cysts (Dandy Walker, } \\
\text { Mega cisterna magna) } \\
\text { Masses: neoplastic and non-neoplastic } \\
\text { Malformations of cortical development, } \\
\text { abnormal migration and proliferation, } \\
\text { heterotopias, abnormal cortical organization }\end{array}$ \\
\hline Vein of Galen aneurismal malformation \\
Increased pressure or ex-vacuo \\
Comalus
\end{tabular}

\section{Brain haemorrhage}

The aetiology of brain haemorrhage varies with age and history. On US a brain haemorrhage appears as a hyperechoic and patchy area that may liquefy and become cystic as it is resorbed. This is how porencephalic cysts form. Ventriculomegely can develop as a complication of an intraventricular bleed and also as a complication of arachnoiditis. US can easily and reliably demonstrate ventriculomegaly. US is also the imaging modality of choice to follow the course of ventriculomegaly.

Germinal matrix bleeds are the least severe of all types (Fig 3). It is classified as a Grade 1 bleed. In preterm infants, before 34 weeks, haemorrhage is seen most frequently in the germinal matrix. The external layer of the cerebellum is a germinal zone as well. (The cerebellum can be demonstrated quite well through the mastoid foramen behind the ear.) After 34 weeks, the germinal matrix involutes. ${ }^{4}$ With intraventricular bleeds, special attention must be given to measuring the
Figure 3: Coronal section of a neonatal cranial ultrasound with a high frequency linear probe, which demonstrates an old, cystic sub-ependymal haemorrhage.

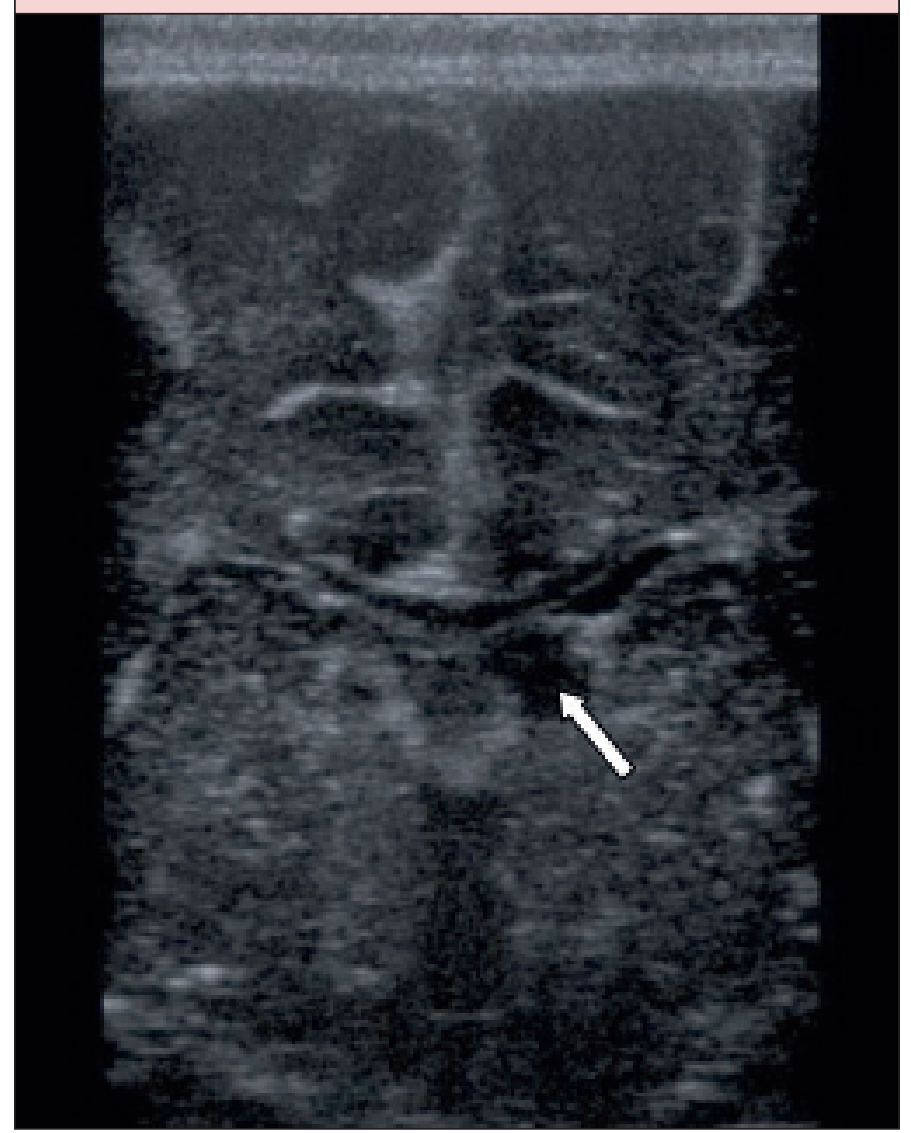

Figure 4: Coronal section. Intraventicular hemorrhage with ventricular dilatation.

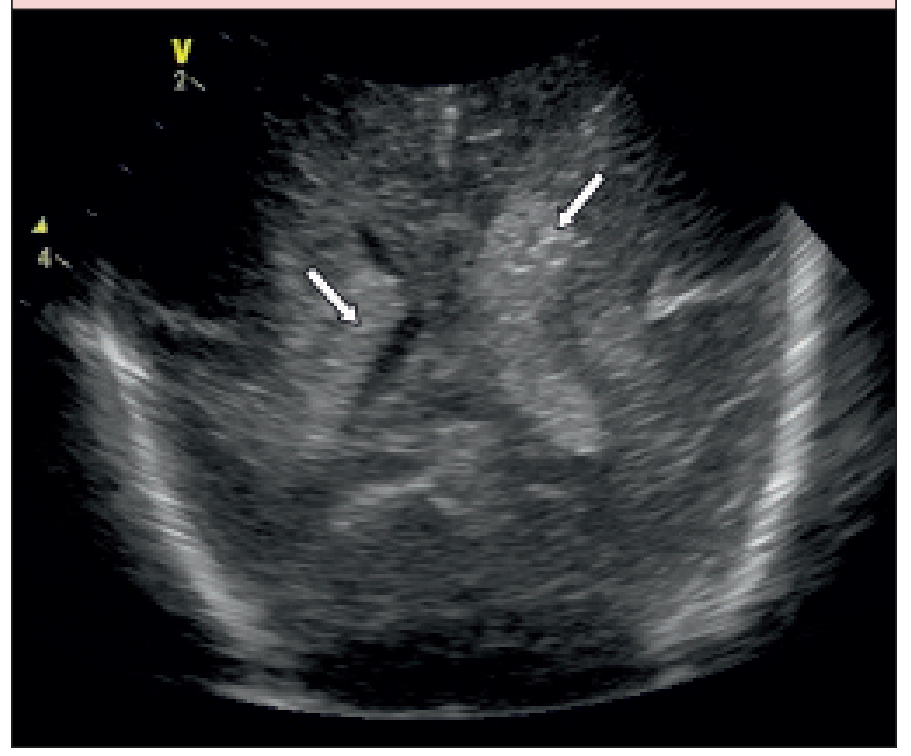

ventricular sizes as it changes the classification. An intraventricular bleed without ventricular dilatation is a Grade 2 bleed, and an intraventricular bleed with ventricular dilatation is a Grade 3 bleed (Fig 4).

Severe hydrocephalus is a complication of an intraventricular bleed, so a base-line ventricular measurement is valuable. Intraparenchymal bleeds, or periventricular haemorrhagic infarctions (PVHI), are the 
worst (Fig 5). The former Grade 4 bleeds, are now classified as Grade 3 + PVHI. ${ }^{1}$ Epidural, subdural and subarachnoid bleeds are rare (Fig 6).

\section{Figure 5: Coronal view. Grade 4 intra parenchymal hemorrhage/Grade 3 with periventricular hemorrhagic} infarction.

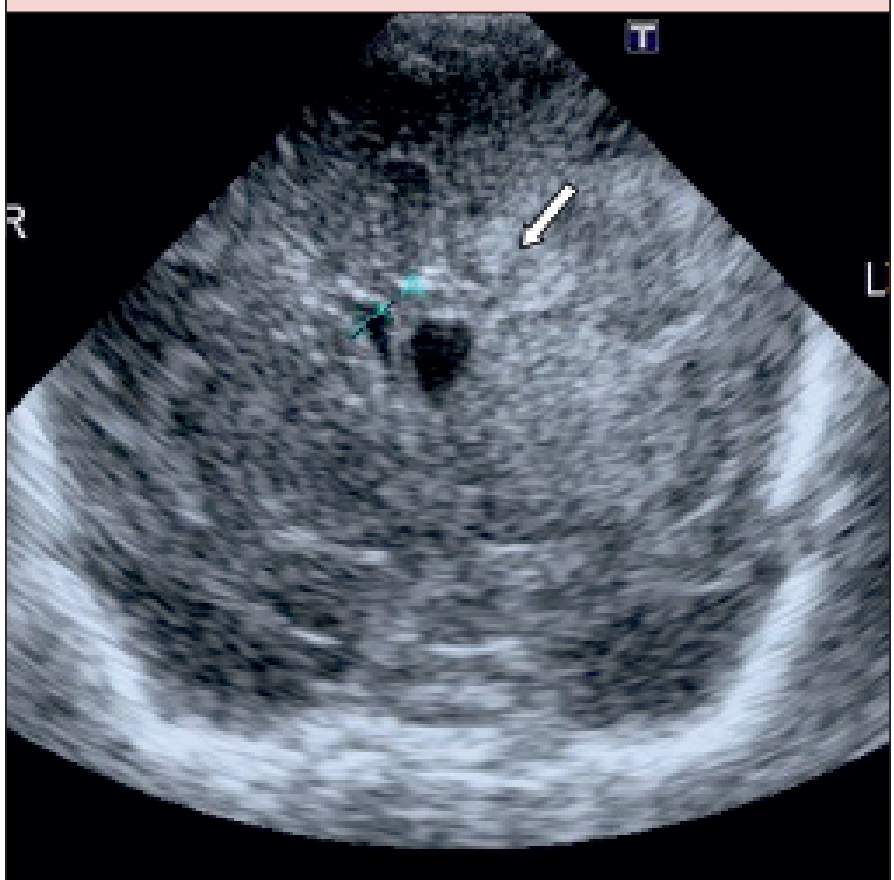

Figure 6: Neonatal skull ultrasound studies in the same patient which demonstrates sub-dural heamorrhage in different stages of evolution. The first image demonstraes a hyperechoic acute haemorrhage. The second image, a week later, demonstrates mixed hyperechoic and hypoechoic fluid components as the haemorrhage changes to an effusion.

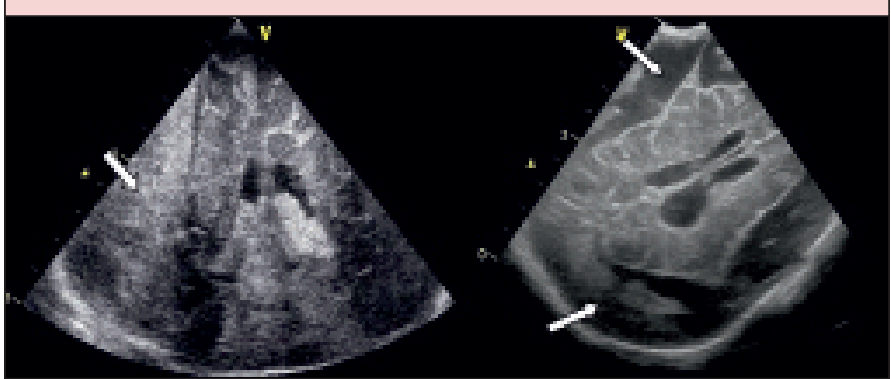

\section{Hypoxic-ischemic-encephalopathy (HIE)}

HIE is a major cause of morbidity. The pattern of brain lesions is related to the state of brain development and maturation. ${ }^{1}$ US cannot detect white matter injury early and reliably. After two to three days, US may only demonstrate a non-specific increase in echogenicity, which is difficult to differentiate from the normal periventricular

hyperechogenicity of prematurity. Periventricular flaring in infants of less than 32 weeks gestation may present ischemic and/or inflammatory damage. ${ }^{5}$ When persisting for over one week, and when the echogenicity exceeds that of choroid plexus, flares are considered the first stage of periventricular leukomalacia. ${ }^{5}$ MRI is the examination of choice for suspected HIE. In the more mature infant, focally increased echogenicity is demonstrated in the thalami and basal ganglia. If it is patchy, non-homogenous and very irregular, white matter hypoxia should be suspected. ${ }^{1}$ Later, cavities develop in the white matter that are easier to demonstrate on US (Fig 7) - especially with a high frequency linear probe. These cysts develop later than the first month after birth and may not be visible any more at 40 weeks post menstrual age.

\section{Figure 7: Coronal section. Periventricular cystic areas.}

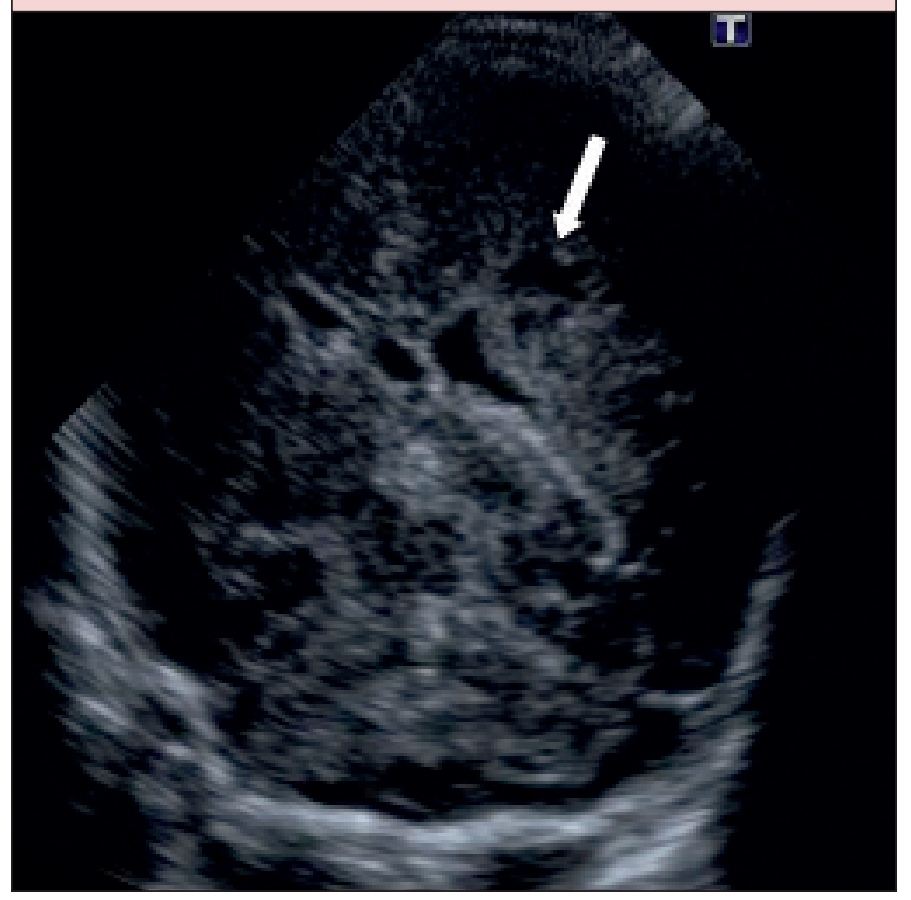

\section{Focal infarction}

Focal infarction usually occurs two to three days after birth and is idiopathic in origin. US may show areas of reduced vascularity at first. ${ }^{1}$ A triangular area of hyperechogenicity may be demonstrated later.

\section{Malformations}

Ventriculomegaly is easy to demonstrate on sonar. Dilated ventricles can be demonstrated on all sonographic views. The lateral, third and fourth ventricles should be examined. The lateral ventricles are demonstrated on the coronal and parasagittal views. The third and the fourth ventricles can be demonstrated on the coronal and sagittal views, and also on transtemporal view. For purposes of repeat US it is important to do standardized measurements.

Some of the brain malformations can be demonstrated on US, but detailed assessment by MRI should follow.

Midline defects can be demonstrated fairly well on US. Although agenesis of the corpus callosum can be diagnosed reliably on sonar, there are often other associated abnormalities that may not be as clear. On US imaging, there is absence of the corpus callosum with widely separated lateral ventricles and large, medially concave occipital horns.

Although ultrasound can diagnose alobar and semilobar holoprosencephaly, it is not as reliable in 
diagnosing lobar and interhemispheric

holoprosencephaly (Fig 8). Alobar holoprosencephaly has a single ventricle, fused thalami and a thin cortical mantle. In semilobar holoprosencephaly, there is a single ventricular body with poorly formed occipital horns, partially separated thalami and more brain parenchyma.

The Chiari malformation is a spectrum of brainstem and cerebellar abnormalities with a small posterior fossa and caudal displacement of the cerebellum. It is associated with meningomyelocele. Repair of the meningomyelocele can increase hydrocephalus.

The Dandy Walker malformation has a large posterior fossa cyst on US, in combination with hypoplasia or agenesis of the cerebellar vermis.

Brain tumors can occasionally be demonstrated on US, but have a poor prognosis.

Vascular malformations like the Vein of Galen malformation, can be seen on grey-scale US with confirmation on color Doppler (Fig 9).

Schizencephaly can give a bizarre image on US with

Figure 8: Corona section. Neonatal cranial ultrasound, coronal section, which demonstrates lobar holoprosencephaly.

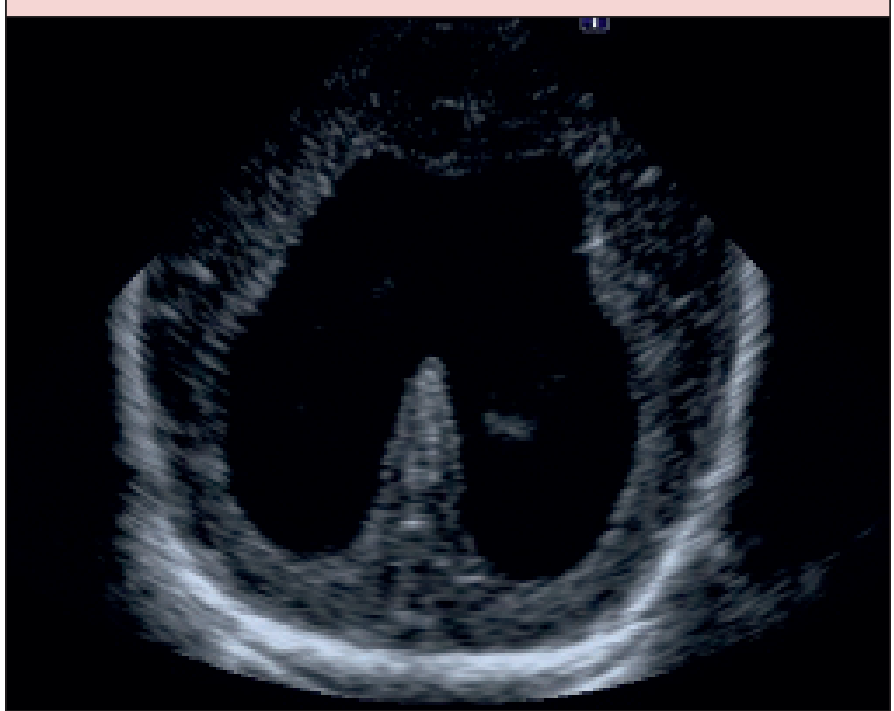

Figure 10: Coronal section. Neonatal cranial ultrasound which demonstrates schizencephaly.

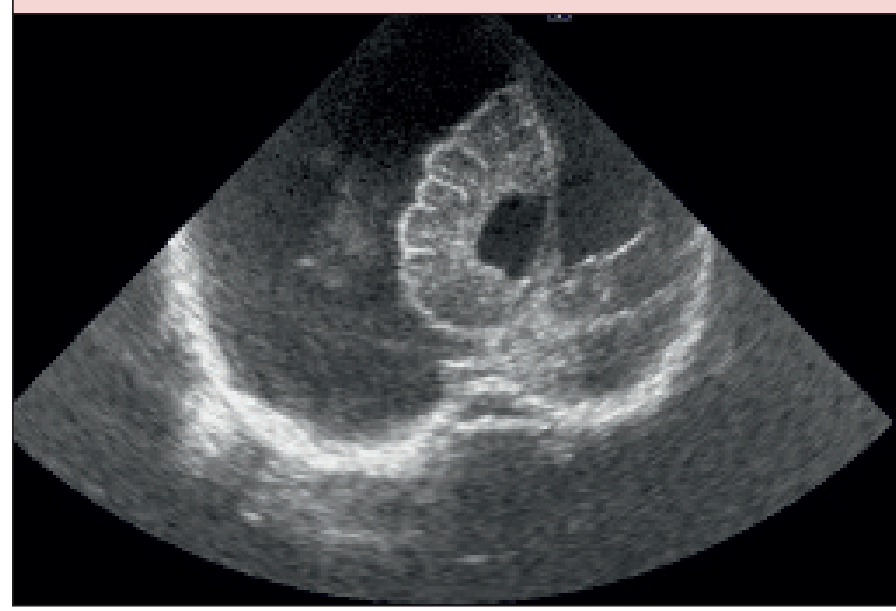

clefts communicating with the ventricles (Fig 10).

Congenital infections by Cytomegalovirus, Toxoplasmosis, Herpes, Rubella, Syphillis and HIV can affect the brain. US can not demonstrate developmental abnormalities and is also poor in outlining the periventricular and basal ganglia calcifications.

Echogenic vasculature/ lenticulostriate or mineralizing vasculopathy, can be seen as linear, branching, echogenic bands in the thalamus and basal ganglia. There is no posterior shadowing and normal flow in the vessels, but it is a non-specific finding

\section{Cardiac Ultrasound}

Indications for cardiac ultrasound in a neonatal unit:

1) Mostly for a Patent Ductus Arteriosus (PDA), to confirm its presence, size and hemodynamic significance. Often it has to be repeated frequently to demonstrate closure with, or without treatment.

2) Confirmation of antenatally detected abnormalities.

3) Cardiac screening when there are other abnormalities, e.g. in chromosomal abnormalities, VACTERL association.

4) To rule out cardiac abnormalities before surgery, e.g. for anus imperforatum.

Figure 9: Coronal section. Vein of Galen malformation with tubulent flow on the Doppler study.

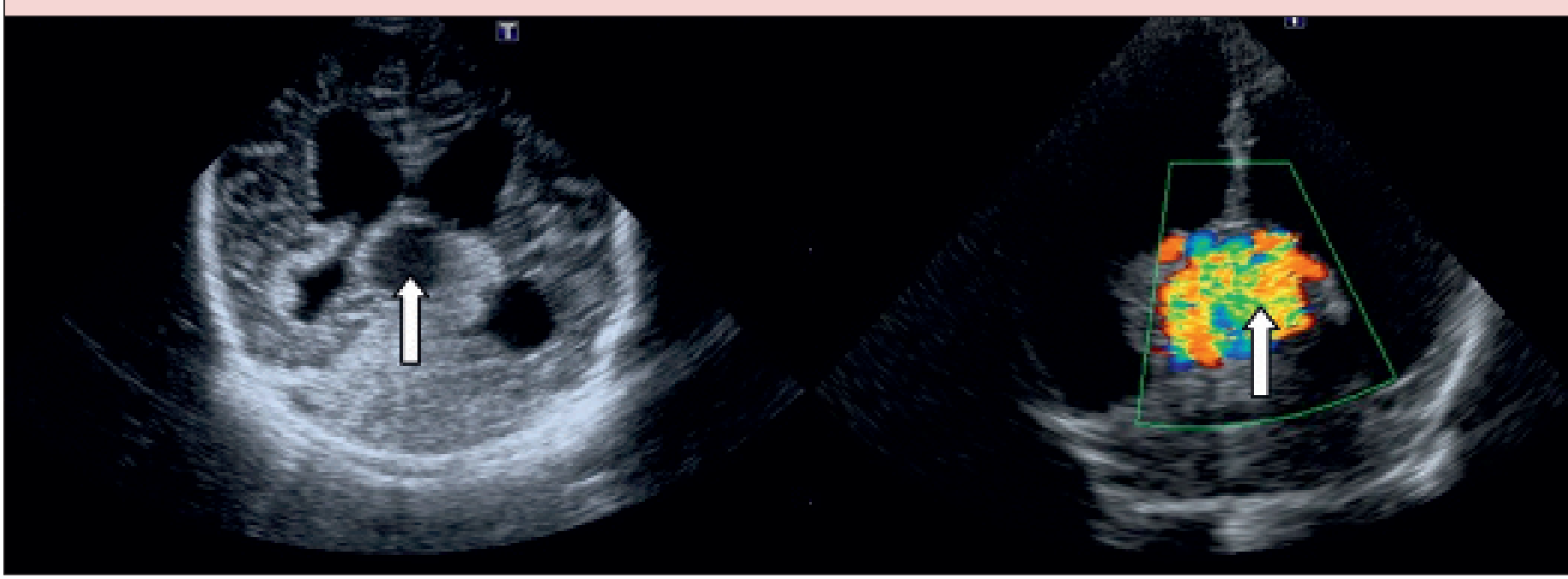


Congenital heart disease (CHD) is a leading cause of death in children with congenital malformations. ${ }^{6}$ Echocardiography is the most important, non-invasive method of diagnosing CHD. It is mainly performed by paediatric cardiologists. But, due to sheer volume of work, paediatric cardiologists can not examine all neonates. Cardiac screening can be performed by paediatricians, neonatologists, radiologists and technicians

PDA screening is vital in a neonatal unit, where PDA is often the only abnormality. A single view can confidently diagnose a PDA (Fig $1 \mathrm{la}$ and b). Use a transverse section, with a view of the descending aorta longitudinally and the left pulmonary artery "blind ending". The PDA demonstrates flow from the descending aorta to the pulmonary artery under normal hemodynamic conditions. With increased pressure in the right heart chambers, PDA may change direction and flow from the pulmonary artery to the aorta. The width of the PDA can be measured accurately and followed up on repeated studies

Figure 11a and b: PDA. Off axis left parasternal view. 11a) Thick arrow - PDA. Short arrow - Pulmonary artery. Long arrow - descending Aorta. 11b) Colour Doppler demonstrates turbulent flow.
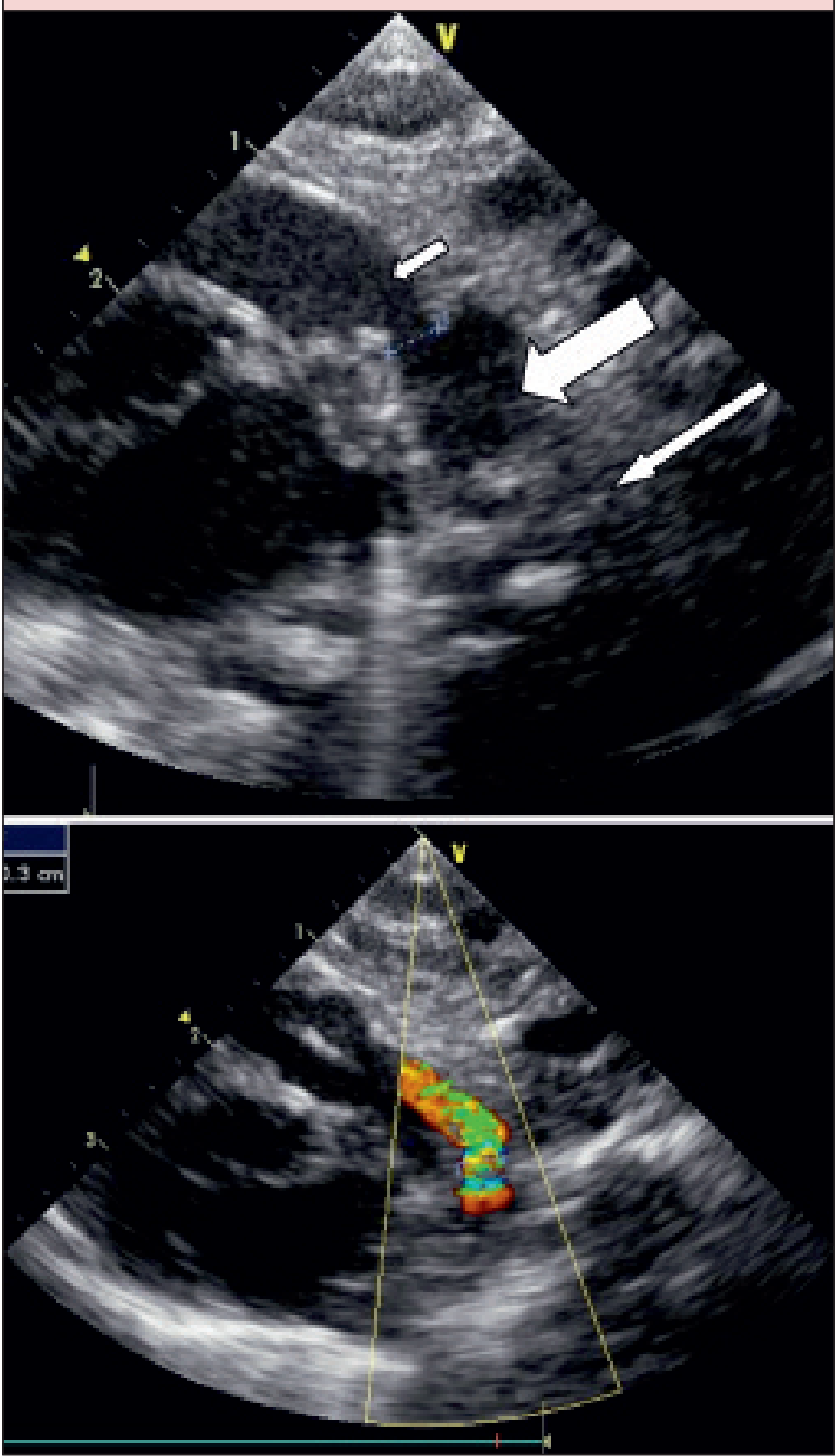

\section{Abdominal ultrasound}

Indications for abdominal ultrasound in a neonatal unit:

1) Confirmation of antenatally detected abnormalities.

2) When there is a palpable mass

3) Screening when there are other abnormalities

4) To determine internal sexual organs in ambiguous genitalia.

A high frequency linear or small curvilinear transducer should be used to examine the neonatal abdomen.

Demonstrate the kidneys, liver, gallbladder and spleen. It is important to look for free fluid. If indicated, the uterus must be examined as well.

Renal abnormalities are the most common abnormalities demonstrated on US, accounting for $20-50 \%$ of all congenital disorders diagnosed prenatally. ${ }^{7}$ Abnormalities range from those that are incompatible with life, to clinically insignificant ones. ${ }^{7}$ Diagnosis is important because early treatment of some conditions may prevent irreversible renal damage. The most common renal abnormality demonstrated on US is dilatation of the renal pelvis. ${ }^{7}$ Other abnormalities that can be demonstrated are: renal agenesis, multicystic dysplasia and polycystic kidneys, abnormal position of the kidney and horseshoe kidney.

When diagnosing hydronephrosis, the dilated calyces must open into the renal pelvis (Fig 12), differentiating it from renal cystic disease, where the cystic areas do not open into the pelvis (Fig 13). Hydronephrosis can be classified as mild when there is pelvic dilatation, but no calyceal dilatation, moderate when there is pelvic and calyceal dilatation and severe if there is loss of renal cortex.

There is no correlation between the degree of pelvicalyceal dilatation and differential renal function. ${ }^{8}$ But poor or loss of cortico-medullary differentiation on US is associated with poor renal function. ${ }^{8}$ For the adequate demonstration of medullary pyramids, a high frequency transducer must be utilized. Small cystic spaces in the medullary pyramids and small cortical cysts are associated with severe abnormal differential renal function. ${ }^{8}$

Most often a palpable mass in a neonate is a kidney. US can differentiate between masses of renal, hepatic, uterine, ovarian, biliary and other origins.

\section{Figure 12: Hydronephrosis. Dilated calyces open into the} dilated renal pelvis

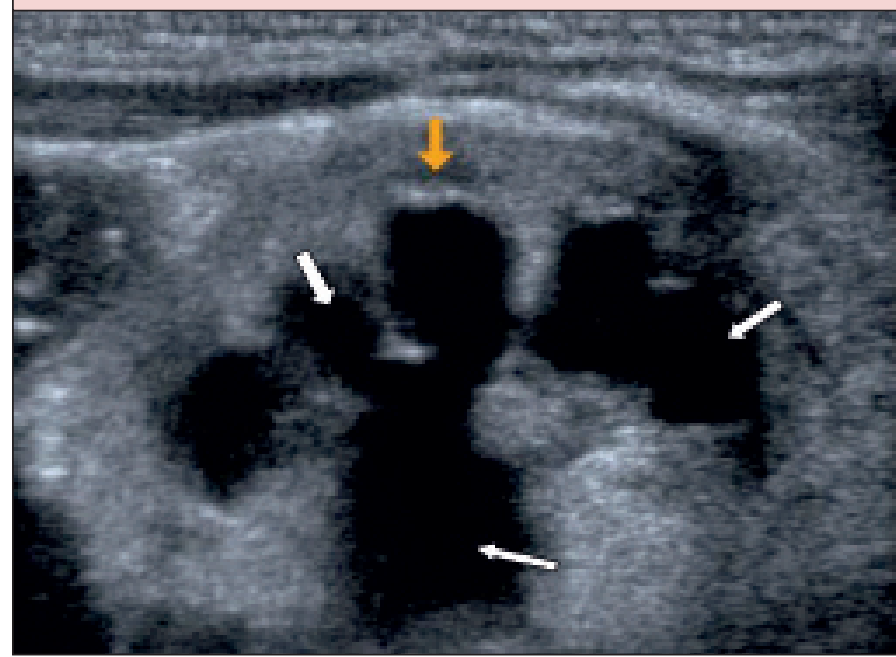




\section{Figure 13: Dysplastic kidney, with multiple cysts of different sizes.}

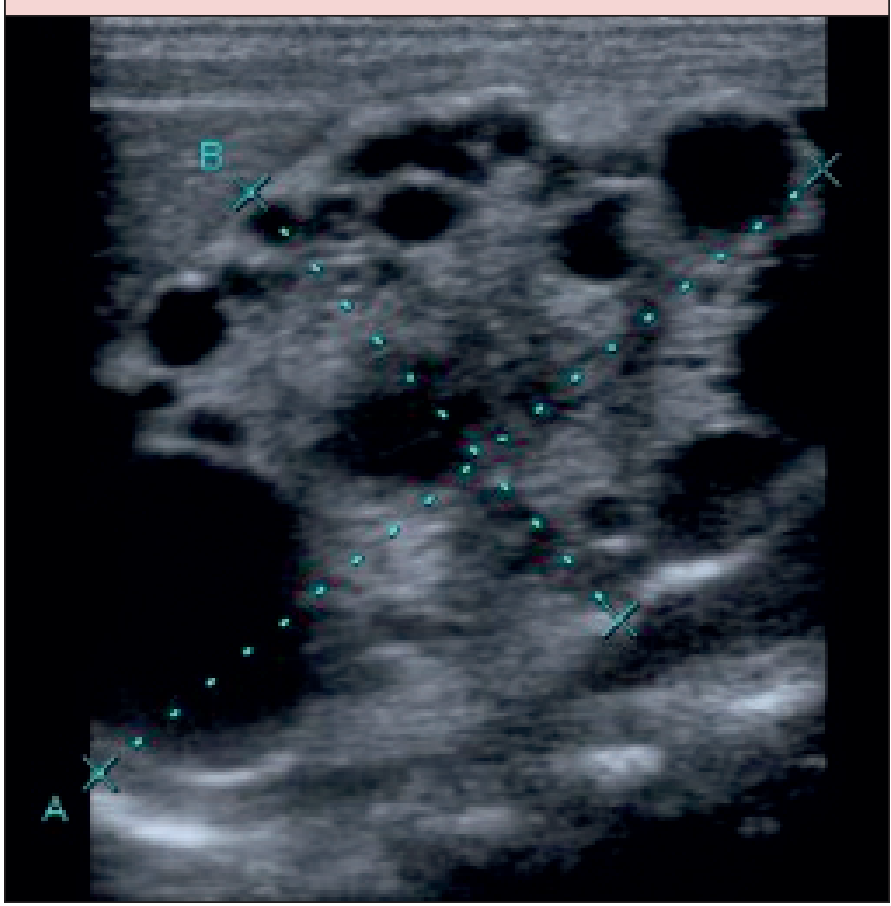

In cases of ambiguous genitalia, it is easier to screen the neonate within days of birth, when the maternal hormones still have an effect on the uterus and it can be demonstrated clearly.

US can give important information in the neonate with necrotizing enterocolitis. A high frequency linear or curvilinear probe should be used. Special attention should be given to the right iliac fossa. US can demonstrate intramural gas, portal venous gas and free intraperitoneal gas like plain abdominal radiography, but in addition, it can also demonstrate intra-abdominal free fluid, bowel wall thickness and bowel wall perfusion. ${ }^{9}$

\section{General precautions in a neonatal unit}

1) The operator must wash her/his hands before the examination

2) Wear protective clothing

3) Clean the transducer.
4) Use only a very small amount of coupling gel. Infants can cool off and collapse in a matter of seconds.

5) Work as fast as possible.

6) Do not apply pressure on the transducer.

7) Ask for help if caps, plasters or tubes must be moved. Try to work around it and not to move the infant if critically ill.

8) It is necessary to use high resolution transducers like the 5-10MHz micro-curved or phased array/sector probes, 7 $12 \mathrm{MHz}$ linear probes.

\section{Conclusion}

US is portable, non-invasive and safe and can give valuable information in a neonatal unit.

\section{References}

1. Simbrunner, J. Riccabona, M. Imaging of the neonatal CNS. European Journal of Radiology.2006;60:133-151.

2. Leijser, Lara M. de Vries, Linda S. Cowan, Frances M. Using cerebral ultrasound effectively in the newborn infant. Early Human Development.2006;82: 827-835.

3. Manual of diagnostic ultrasound. Edited by PES Palmer. Published by the World Health Organization in collaboration with the World Federation for Ultrasound in Medicine and Biology. 1995.

4. Yikilmaz A, Taylor GA. Cranial sonography in term and near-term infants. Pedatr Radiol.2008;38:605-16. (2008) 38:605-616.

5. Leisjer LM, Liauw L, Veen S, De Boer IP, Walther F, Van WezelMeijler. Comparing brain white matter on sequential cranial ultrasound and MRI in very preterm infants. Neuroradiology.2008;50:799-811

6. Schweigmann G Gassner I Maurer K. Imaging the neonatal heartEssentials for the radiologist. European Journal of Radiology.2006;60:159-170

7. Hálek J, Flögelová H Michálková K. Old ich S, Lubomír DLubomir Z, et al, Jan Diagnostic accuracy of postnatal ultrasound screening for urinary tract abnormalities. Pediatric Nephrology.2010;25:281-287.

8. Chavhan G Daneman AMoieddin RLim RLanglois V Ttraubici J Renal pyramid echogenicity in ureteropelvic junction obstruction: correlation between altered echogenicity and differential renal function. Pediatric Radiology2008;38:1068-73.

9. Epelman M. Daneman A Navarro O Mmorag I Moore A, M. Kim, Jae Hong, et al. Necrotizing Enterocolitis: Review of State-of the-Art Imaging Findings with Pathologic Correlation. RadioGraphics. 2007; $27: 285-305$ 